\title{
A Study on the Effectiveness of Video Call Dispatcher-Assisted Cardiopulmonary Resuscitation in Enhancing the Quality of Cardiopulmonary Resuscitation Among Laymen Bystanders in Malaysia
}

\author{
(1) Mohamad Faizal Zulkhifli, (1) Rashidi Ahmad, (1) Mohd Zahir Amin Mohd Nazri, (1) Abdul Muhaimin Noor Azhar
}

Department of Emergency Medicine, Faculty of Medicine, University of Malaya, Kuala Lumpur, Malaysia

\begin{abstract}
Aim: Technology has enabled the utilization of dispatcher-assisted cardiopulmonary resuscitation (DA-CPR) through mobile video call for layman bystanders as opposed to the conventional audio call. This study aimed to examine the effects of medical guidance through a video call on cardiopulmonary resuscitation (CPR) quality during the management of cardiac arrest.

Materials and Methods: A quasi-experimental study was conducted on 102 participants. These participants were divided into two groups: laymen who received CPR instructions through dispatcher-assisted video call $(n=51)$ and healthcare personnel $(n=51)$ who did not receive any assistance. The compression rate and depth, compression fraction, chest recoil, and hand positioning were compared between the groups.

Results: Laymen who received video call DA-CPR performed better than the healthcare personnel in adhering to the recommended compression rate (109.7 $\mathrm{min}^{-1}$ vs $\left.126.7 \mathrm{~min}^{-1}, \mathrm{p}<0.05\right)$ and allowing chest recoil $(94.8 \%$ vs $84.4 \%, p<0.05)$ but performed poorer in terms of compression depth $(58.8 \%$ vs $98 \%, p<0.05)$ and chest compression fraction $(49.2 \%$ vs $60.8 \%, p<0.05)$. Hand positioning in the layman group was less accurate in comparison with that in the healthcare personnel group (90.2\% vs $96.1 \%)$, but the result was not statistically significant $(\mathrm{p}=0.24)$.

Conclusion: In this study, video call DA-CPR allows laymen to deliver CPR with better compression rate, chest recoil, and hand placement than did healthcare personnel. However, inadequate compression depth and low chest compression fraction in the video call DA-CPR group show that more study is needed to deliver effective instructions during the video call to improve these areas.
\end{abstract}

Keywords: Dispatcher-assisted, video call, cardiopulmonary resuscitation, out of hospital cardiac arrest, mobile phone

\section{Introduction}

Out of hospital cardiac arrest (OHCA) is one of the most highlighted areas when discussing cardiopulmonary resuscitation (CPR). According to the American Heart Association (AHA), the survival rate of OHCA was $10-12 \%$ (1). A strong chain of survival has been shown to improve the chances of survival and recovery for cardiac arrest victims. Modifiable factors associated with increased OHCA survival includes early bystander CPR, Emergency medical service (EMS) response times, and application of an automated external defibrillator in communities (2). These factors represent opportunities for improvements in saving lives.

In the event of OHCA, prompt attendance by medical personnel trained in basic life support (BLS) or Advance Cardiac Life Support (ACLS) would be an ideal situation. However, in Malaysia, the average time taken for the ambulance to arrive at the scene is 19 minutes (3). Thus, to improve survival in OHCA, early bystander CPR is imperative (4). Regrettably, the rate of bystander CPR in developing nations is still relatively low at $10 \%$ and the quality 
of CPR is in doubt (5). Dispatcher-assisted CPR (DA-CPR) is an advocated solution. Dispatcher protocols designed to improve rapid recognition of arrest and coach CPR may increase survival by reducing preventable time delays to start of chest compressions and improving the quality of bystander CPR (6). Traditional communication via verbal instructions over the phone has a few limitations such as time delay due to the rescuer's conversation with the dispatcher and the inability for the dispatcher to receive real-time visual feedback of the rescuer during CPR (7).

DA-CPR in Malaysia is still in infancy. Lack of resources and financial constraints may affect their progress. With the advancement of mobile technology and widespread internet connectivity, video calls via smartphones have been highly plausible (8). Interactive audio-visual communication is doable. In addition to removing visual barriers that existed in the conventional audio calls, video communication offers realtime audio-visual feedback for both interactive parties, thus improving effective communication. One previous study that examined the differences in the quality of dispatcher-assisted chest compression between video coaching and audio coaching showed that the video coaching group achieved better results in terms of the compression rate, the accuracy of the compression site, and the minimization of the hands-off time (9). Additional benefits of video call DA-CPR include an enhancement in lay rescuer's confidence level, a positive effect on dispatcher's attitude, an improvement of chest compression rate and better airway manoeuvres (10-13).

Based on the current situation in Malaysia, the present study aimed to observe the efficacy of direct medical guidance through a video call during the management of OHCA. We hypothesized that the quality of CPR performed by laymen bystanders who received CPR instructions via video call communication is better than the quality of CPR performed by health care personnel (HCP) bystanders.

\section{Materials and Methods}

\section{Study Design and Setting}

This quasi-experimental study was conducted in the Emergency Department (ED) of the University Malaya Medical Centre over a span of 6 months duration. This study was approved by the Medical Research Ethics Committee of University Malaya (no: no: 2018829-6632, date: 01.02.2019).

\section{Population and Sample Size}

There were two groups involved in this non-randomized pre and post interventional study, i.e., laymen bystanders and HCP bystanders group. Those laypeople who accompanied their parent, spouse, relative or friend at the non-critical zone of ED and who had no CPR training before (laymen bystanders) and HCP bystanders in ED (nurses, medical assistants, and doctors) with a valid BLS certificate were included in the study. The participant who had any physical disability or audio-visual impairment was excluded from the study. The whole idea of this study was to evaluate the CPR performance of laymen bystanders after receiving step-by-step guidance via video call from trained dispatcher. Those certified advanced cardiovascular life support (ACLS) instructor among the emergency residents were appointed as the dispatcher. To prove our hypotheses, the estimated sample size was calculated based on $\alpha$ precision of 0.05 , a two-sided test, and an assumption of moderate effect. Our power calculation was performed in G-Power 3.1.3 software. Hundred and two participants ( $n=51$ in each group) were required to make this study $80 \%$ power with a 5\% significance level.

\section{Materials and Procedures}

During the early stages of the study, we explained the study objective, design, and procedure to those interested laypersons. Written informed consent was obtained once they clearly understood and agreed to take part in the study.

We positioned the simulation CPR mannequin (Resuci Anne QCPR, Laerdal, Stavanger, Norway) and laymen bystanders, in the middle of the room. Samsung Note Fan Edition mobile phone was fixed to a tripod and positioned beside the mannequin. To measure the quality of CPR, QCPR Instructor Apps (version 1.11.21) was connected to the mannequin via Bluetooth connectivity. The dispatcher was provided with an iPhone 8 and was stationed in a separate building.

During the second phase of the study, video calls between the dispatcher and the layman bystanders were made using the WhatsApp Messenger application (version 2.19.81) via the $3 G$ network. Once they got connected, the dispatcher directed the laymen bystanders to perform CPR steps for 2 minutes according to the modified call card instruction module adapted from the national Malaysian Emergency Medical Dispatch protocol (AMPDS $^{\circledR}$ v13.0, MAL-std). Similarly, HCP bystanders were asked to perform CPR on the same training mannequin as per the BLS algorithm but without any guidance or feedback.

\section{Data Collection and Outcome}

The quality of CPR performed by both groups was measured based on the criterion recommended by the 2015 AHA guidelines (14).

The measured variables included:

1. Chest compression rate.

2. Compression depth. 
3. Adequacy of chest recoil.

4. Correct hand placement in the mid sternum area.

5. Chest compression fraction (CCF)

We transferred all the collected data from the QCPR Instructor Apps into the collecting sheet then into the Google Sheet that was accessible via the investigator's Google Drive only. A study participant reference number was used to recognize the participants. All information and data collected either electronically or in the form of hard copy were destroyed after the storage duration unless required otherwise by the ethics committee, regulatory bodies, or institutional bodies.

\section{Statistical Analysis}

Participants who did not meet the inclusion criteria were excluded from statistical analyses. Statistical analysis was performed using Statistical Package for the Social Science (SPSS) version 21.0 (IBM enterprise). Numerical data were reported as mean (SD). Categorical data were reported as a percentage (\%). According to the AHA Guideline 2015, the appropriate chest compression per minute, compression depth $(\mathrm{cm})$ and chest compression fraction are 100-120, 5.0-6.0, and more than 60\%, respectively.

Chi-square test was applied to determine the relationship between laymen bystanders and HCP bystanders chest compression rate and depth. The $p<0.05$ indicates that these variables are not independent of each other and that there is a statistically significant relationship between the categorical variables.

Two-tailed unpaired t-test was conducted within each group to determine the mean difference in the quality of CPR measurements and its significance. In the initial phase, The Kolmogorov-Smirnov normality test was applied to determine the distribution of data. i.e., normal, or abnormal data distribution. $p$-value of $<0.05$ means, the difference between the two samples is significant enough to suggest that the data do not follow a normal distribution. For analysis of normal distribution unpaired data, an independent t-test was applied. Before interpreting the data, Levene's test was conducted to look for homogeneity in the t-test. The differences are declared statistically significant if $\mathrm{p}<0.05$.

\section{Results}

\section{Demographic Characteristics}

A total of 102 volunteers took part in the study. There were 51 participants in each group (Table 1).

The mean age (SD) of laymen bystanders and HCP bystanders were 29.4 (6.6) and 28.1 (4.0) years old, respectively. The predominant gender was female participants. All HCP bystanders were either diploma or degree holder. In comparison, only $75 \%$ of laymen bystanders had higher education background.

\section{High-Quality CPR Performance}

Over the two minutes, CPR process, the mean compression rate (SD) among the laymen bystanders that were guided by video call and among the HCP bystanders were 109.7 (19.1) $\mathrm{min}^{-1}$ and 126.7 (16.5) $\mathrm{min}^{-1}$, respectively (Table 2). Astonishingly, more than half of laymen bystanders (51.0\%) achieved a proper chest compression rate compared to HCP bystanders (37.4\%). Statistically, there was no significant relationship exists between laymen bystanders CPR that were assisted with video call and HCP bystanders in performing appropriate chest compression rate, $X^{2}(1, n=102)=1.95, p=0.16$.

Most of HCP bystanders executed an adequate depth of chest compression compared to the laymen bystanders $(98.0 \%$ vs $58.8 \%, p<0.05)$. Statistically, there was a significant relationship exists between laymen bystanders who performed CPR under video call guidance and HCP bystanders in performing adequate depth of chest compression, $X^{2}(1, n=102)=23.18, p<0.05$.

Table 1. Baseline characteristics of participants from the two study groups

\begin{tabular}{|c|c|c|}
\hline & Laymen bystanders & HCP bystanders \\
\hline \multicolumn{3}{|l|}{ Gender } \\
\hline Male, n (\%) & $25(49)$ & $20(39.2)$ \\
\hline Mean age (SD), years & $29.4(6.6)$ & $28.1(4.0)$ \\
\hline \multicolumn{3}{|l|}{ Level of education } \\
\hline Diploma, n (\%) & $6(11.8)$ & $28(54.9)$ \\
\hline Degree, n (\%) & $26(51.0)$ & $23(45.1)$ \\
\hline Postgraduate, n (\%) & $6(11.8)$ & 0 \\
\hline
\end{tabular}


In terms of correct hand placement during chest compression, both groups (laymen bystanders and HCP bystanders) compressed the chest appropriately at the centre of the chest (90.2\% vs 96.1\%), $X^{2}(1, n=102)=1.38, p=0.24$.

Regarding allowing complete recoil during chest compression, the video call assisted layman bystanders performance was better than HCP bystanders [94.8\% vs $84.4 \%, X^{2}(1, n=102)=7.14$, $\mathrm{p}<0.05)$.

Independent t-test analysis of chest compression rate demonstrated a significant mean difference in chest compression rate between video call guided laymen bystanders CPR and HCP bystanders, t $(100)=4.80, p<0.05$ (Table 3 ). There was also a significant mean difference of chest compression fraction between video call guided laymen bystanders CPR and HCP bystanders, $\mathrm{t}(100)=7.43, \mathrm{p}<0.05$.

\section{Discussion}

This study demonstrated that in general, the HCP bystanders' compression rate was faster than the recommended compression rate (100-120 $\left.\mathrm{min}^{-1}\right)$. This may be because most of the healthcare worker had not gone for any BLS or ACLS refreshment course for 1 year. This may lead to a decline in memory retention and skill set. Interestingly, about 50\% of laymen bystanders with video call assistance performed an appropriate compression rate. Our finding was similar to other studies conducted by Yang et al. (15) and Lee et al. (9). Yang et al. (15) did a comparison study between interactive video call against audio call on the quality of dispatcher-assisted compression-only CPR. The study reported that $46.5 \%$ of the lay rescuers with video call guidance had performed chest compression at an appropriate rate. Lee et al. (9) examined the effect of cellular video phone demonstration on the quality of hands-only CPR compared to audio call. The study showed that $59 \%$ of the test subjects performed the chest compression according to the recommended compression rate. However, the mean compression rate in Yang et al. (15) and Lee et al. (9) studies were $95.5 \mathrm{~min}^{-1}$ and $99.5 \mathrm{~min}^{-1}$, respectively. The fact that the video call in their studies was pre-recorded and could not provide live feedback on the quality of compression rate to the rescuer while the CPR was ongoing may be the main

Table 2. The quality of CPR between laymen bystanders with video call DA-CPR guidance and healthcare personnel bystanders

\begin{tabular}{|l|l|l|}
\hline Quality of CPR & Laymen bystanders & HCP bystanders \\
\hline Mean compression, rate/min (SD) & $109.7(19.1)$ & $126.7(16.5)$ \\
\hline Allow complete chest recoil, (\%) & 94.8 & 84.4 \\
\hline Mean chest compression fraction, (\%) & 49.2 & 60.8 \\
\hline Correct hand placement, (\%) & 90.2 & 96.1 \\
\hline DA-CPR: dispatcher-assisted CPR, CPR: Cardiopulmonary resuscitation, HCP: Health care personnel, SD: Standard deviation \\
\hline
\end{tabular}

Table 3. Comparison of two independent samples (chest compression rate and chest compression fraction between laymen bystanders with video call DA-CPR guidance and healthcare personnel bystanders

\begin{tabular}{|c|c|c|c|c|c|c|c|c|c|c|}
\hline & & \multicolumn{2}{|c|}{$\begin{array}{l}\text { Levene's test } \\
\text { for equality of } \\
\text { variances }\end{array}$} & \multicolumn{7}{|c|}{ t-test for equality of means } \\
\hline \multicolumn{11}{|c|}{$\begin{array}{l}\text { 95\% confidence } \\
\text { interval of the } \\
\text { difference }\end{array}$} \\
\hline & & $\mathrm{F}$ & Sig. & $\mathrm{t}$ & df & $\begin{array}{l}\text { Sig. } \\
\text { (2-tailed) }\end{array}$ & $\begin{array}{l}\text { Mean } \\
\text { difference }\end{array}$ & $\begin{array}{l}\text { SE } \\
\text { difference }\end{array}$ & Lower & Upper \\
\hline \multirow{2}{*}{$\begin{array}{l}\text { Chest } \\
\text { compression } \\
\text { rate per } \\
\text { minute }\end{array}$} & $\begin{array}{l}\text { Equal variances } \\
\text { assumed }\end{array}$ & 0.018 & 0.893 & 4.802 & 100 & 0.000 & 16.980 & 3.536 & 9.965 & 23.996 \\
\hline & $\begin{array}{l}\text { Equal variances } \\
\text { not assumed }\end{array}$ & & & 4.802 & 97.834 & 0.000 & 16.980 & 3.536 & 9.963 & 23.998 \\
\hline \multirow{2}{*}{$\begin{array}{l}\text { Chest } \\
\text { compression } \\
\text { fraction (\%) }\end{array}$} & $\begin{array}{l}\text { Equal variances } \\
\text { assumed }\end{array}$ & 0.392 & 0.533 & 7.428 & 100 & 0.000 & 11.588 & 1.560 & 8.493 & 14.683 \\
\hline & $\begin{array}{l}\text { Equal variances } \\
\text { not assumed }\end{array}$ & & & 7.428 & 99.983 & 0.000 & 11.588 & 1.560 & 8.493 & 14.683 \\
\hline
\end{tabular}


reason why few of the laymen bystanders did not perform well as expected. In contrast, the mean chest compression rate in our study was $110 \mathrm{~min}^{-1}$. Most probably, the higher CPR rate in our study was attributed to the live feedback by the dispatchers. The dispatcher had instructed the lay rescuer to compress faster in synchrony with his counting whenever the dispatcher realized the rescuer was compressing too slow.

Our study demonstrated more subjects in the HCP bystanders group achieved appropriate compression depth (92.7\%) compared to the video call assisted laymen bystanders group (57.5\%). Bolle et al. (16) examined CPR quality between a video call and audio call among laymen bystander. The study revealed that the average chest compression depth performed by the participants in the video group was $3.7 \mathrm{~cm}$ with only $35 \%$ of the test subjects in the video group performed adequate compression depth (16). In another study conducted by Yang et al. (15) the researchers discovered that the video group subjects had a mean chest compression depth of $3.6 \mathrm{~cm}$ with only $20 \%$ of them achieved an adequate compression depth. Offering feedback on compression depth to the rescuer is extremely difficult as there is no way for the dispatcher to objectively evaluate the compression depth. Hence, the dispatcher is advisable to accentuate on "push hard" on every CPR cycle, and it does not matter whether the chest compression depth is adequate or not to ensure adequate compression depth is provided.

In addition to proper compression rate and compression depth, rescuers must also minimise interruption during chest compression to achieve high-quality CPR. The AHA guidelines 2015 recommends chest compression fraction (defined as the percentage of total resuscitation time that compressions are performed) of more than $60 \%$ as a measure of minimal interruption during chest compression (14). This study demonstrated that the mean chest compression fraction of video call assisted laymen bystanders did not accomplish the suggested parameter (49.2\%) and mean chest compression fraction of HCP bystanders was acceptable though marginal result (60.8\%). From our observation, layman rescuers took much longer time to open the airway and to ensure adequate rescue breaths were provided during the video call.

Regarding the correct hand placement during chest compression, the performance of laymen bystanders group was comparable to the HCP bystanders group (90.2\% vs $96.1 \%)$. Similar findings were noted from Yang et al. (15) and Lee et al. (9) studies whereby the percentage of correct hand placement in their video call group were $84 \%$ and $71.8 \%$, respectively. These results may be attributed to the fact that video call allows real-time visual feedback to the dispatcher and thus, can troubleshoot incorrect hand placement immediately.
We have no issue in claiming that video call assisted CPR expedites the initiation of life-saving intervention but we are still unsure the overall quality of CPR performed by naïve laymen bystanders that is guided by video call. However, we are very positive that video call assisted CPR may enhance the quality of CPR among CPR trained laypersons.

\section{Study Limitations}

There are a few limitations in this study. Firstly, this study was performed in a simulated cardiac arrest situation where other factors that may affect CPR performance such as prolonged CPR while waiting for EMS arrival, anxiety and stress are absent. Secondly, the mobile phone used in our study was placed in a fixed position with a tripod to maximise the audio-visual quality. In real life situation where there is only one rescuer who would make the phone call and perform CPR, it may be challenging to find a proper spot to place the mobile phone whilst simultaneously doing CPR and maintaining optimal audiovisual communication. Finally, the dispatcher used a modified dispatch protocol designed for audio DA-CPR. The development of a validated video call dispatch protocol in the future may elucidate the benefit of video call DA-CPR.

\section{Conclusion}

The majority of OHCA occurs in a public setting. Bystander CPR offers many survival advantages. Immediate CPR can double or triple chances of survival after sudden cardiac arrest. The chance of survival is higher if laymen bystanders provide high-quality CPR before the arrival of the EMS team. Here in our study we concluded that video call assisted CPR may enhance the quality of CPR provided it can evaluate the laymen bystanders performance and at the same time it can provide prompt feedback related to CPR interventions.

\section{Ethics}

Ethics Committee Approval: This quasi-experimental study was conducted in the Emergency Department of the University Malaya Medical Centre over a span of 6 months duration. This study was approved by the Medical Research Ethics Committee of University Malaya (no: 2018829-6632, date: 01.02.2019).

Informed Consent: Written informed consent was obtained once they clearly understood and agreed to take part in the study.

Peer-review: Externally peer-reviewed.

\section{Authorship Contributions}

Concept: M.Z.A.M.N., A.M.N.A., Design: M.Z.A.M.N., A.M.N.A., Data Collection or Processing: M.F.Z., Analysis or Interpretation: M.F.Z., R.A., M.Z.A.M.N., A.M.N.A., Literature Search: M.F.Z., R.A., Writing: M.F.Z., R.A. 
Conflict of Interest: No conflict of interest was declared by the authors.

Financial Disclosure: The authors declared that this study received no financial support.

\section{References}

1. Roger VL, Go AS, Lloyd-Jones DM, Adams RJ, Berry JD, Brown TM, et al. Heart disease and stroke statistics--2011 update: a report from the American Heart Association. Circulation. 2011;123:e18-209.

2. Bjørshol CA, Søreide E. Improving survival after cardiac arrest. Semin Neurol. 2017;37:25-32.

3. Nik Him NAS, Hussin K, Hassan MR, Hamzah ML, Fadzli A, Nik Mohamad NA. Ambulance Response Time at Three Selected Tertiary Hospitals in Malaysia: Performance of Hospital-Based Ambulance Equipped with Computerized Call Centre System. Mal J Appl Sci. 2018;3:42-50.

4. Nielsen AM, Isbye DL, Lippert FK, Rasmussen LS. Can mass education and a television campaign change the attitudes towards cardiopulmonary resuscitation in a rural community? Scand J Trauma Resusc Emerg Med. 2013;21:39.

5. Song KJ, Oh DJ. Current status of CPR in Korea. Korean J Med. 2007;73:4-10.

6. Rea TD, Eisenberg MS, Culley LL, Becker L. Dispatcher-assisted cardiopulmonary resuscitation and survival in cardiac arrest. Circulation. 2001;104:2513-6.

7. Merchant RM, Abella BS, Abotsi EJ, Smith TM, Long JA, Trudeau ME, et al. Cell phone cardiopulmonary resuscitation: audio instructions when needed by lay rescuers: a randomized, controlled trial. Ann Emerg Med. 2010;55:53843.e1.

8. Suruhanjaya Komunikasi Dan Multimedia Malaysia, Malaysian Communications and Multimedia Commission. Hand phone users survey 2017, statistical brief number twenty-two. In: Commission MCaM, editor. Cyberjaya, Malaysia 2017. Available at: https://www.skmm.gov.my/ skmmgovmy/media/General/pdf/HPUS2017.pdf
9. Lee JS, Jeon WC, Ahn JH, Cho YJ, Jung YS, Kim GW. The effect of a cellularphone video demonstration to improve the quality of dispatcher-assisted chest compression-only cardiopulmonary resuscitation as compared with audio coaching. Resuscitation. 2011;82:64-8.

10. Bolle SR, Johnsen E, Gilbert M. Video calls for dispatcher-assisted cardiopulmonary resuscitation can improve the confidence of lay rescuers-surveys after simulated cardiac arrest. J Telemed Telecare. 2011;17:88-92.

11. Johnsen E, Bolle SR. To see or not to see--better dispatcher-assisted CPR with video-calls? A qualitative study based on simulated trials. Resuscitation. 2008;78:320-6.

12. Lin YY, Chiang WC, Hsieh MJ, Sun JT, Chang YC, Ma MH. Quality of audio-assisted versus video-assisted dispatcher-instructed bystander cardiopulmonary resuscitation: A systematic review and meta-analysis. Resuscitation. 2018;123:77-85.

13. Yang CW, Wang HC, Chiang WC, Chang WT, Yen ZS, Chen SY, et al. Impact of adding video communication to dispatch instructions on the quality of rescue breathing in simulated cardiac arrests--a randomized controlled study. Resuscitation. 2008;78:327-32.

14. Kleinman ME, Brennan EE, Goldberger ZD, Swor RA, Terry M, Bobrow BJ, et al. Part 5: Adult Basic Life Support and Cardiopulmonary Resuscitation Quality: 2015 American Heart Association Guidelines Update for Cardiopulmonary Resuscitation and Emergency Cardiovascular Care. Circulation. 2015;132(18 Suppl 2):S414-35.

15. Yang CW, Wang HC, Chiang WC, Hsu CW, Chang WT, Yen ZS, et al. Interactive video instruction improves the quality of dispatcher-assisted chest compression-only cardiopulmonary resuscitation in simulated cardiac arrests. Crit Care Med. 2009;37:490-5.

16. Bolle SR, Scholl J, Gilbert M. Can video mobile phones improve CPR quality when used for dispatcher assistance during simulated cardiac arrest? Acta Anaesthesiol Scand. 2009;53:116-20. 